\title{
Finanzpolitik und Steuerverwaltung in Tansania während der "verlorenen Entwicklungsdekade"
}

\author{
Von Ulrich Fanger
}

Tansania hat seit der Arusha-Erklärung (1967) einen Weg des eigenständigen Sozialismus nach dem Prinzip der "self-reliance" verfolgt, der auf Erschließung der eigenen Fähigkeiten in größtmöglicher Unabhängigkeit von Außenressourcen und weitgehenden Abbau der innergesellschaftlichen Wohlstands- und Statusunterschiede abzielte. Schlüsselbereiche der Entwicklungspolitik waren der Ausbau des Gesundheits- und Bildungswesens sowie die ländliche Siedlungsreform (Dorfentwicklung / Siedlungskonzentration / Ujamaa), durch die ab 1975 der Landwirtschaftsanbau kollektiviert wurde.1

Unbestreitbaren sozialen Errungenschaften (z.B. den hohen Scholarisierungs- und Alphabetisierungsraten) steht jedoch eine krisenhafte Entwicklung der industriellen und landwirtschaftlichen Produktivität und der Selbstversorgungsbasis gegenüber, die sich seit Mitte der siebziger Jahre laufend verschärft hat. Wesentliche endogene Faktoren dieser Entwicklung waren: der wachsende latente Widerstand der Landbevölkerung angesichts der zunehmenden Fehlsteuerung der Agramarkt- und Preispolitik, die disfunktionale Ausweitung der staatlichen Industrieregie, Úberbürokratisierung in allen Bereichen sowie die Kosten des militärischen Engagements in Uganda. Sie haben die wirtschaftliche Leistungsfähigkeit des Landes strukturell geschwächt. Als entscheidende außenwirtschaftliche Einflüsse lösten ab 1974 die Energiepreiskrise (Verteuerung von Energieträgern und Düngemitteln etc.) und die Verschlechterung der Austauschbedingungen für tansanische Exportgüter einen negativen Wirkungskreislauf in Gang, der zu wachsender Abhängigkeit von Entwicklungshilfeleistungen, massiver Außenverschuldung und zunehmender Abkapselung von Auslandsimporten fuhrte. Tansania war in den siebziger Jahren das afrikanische Land mit dem höchsten Wirtschaftshilfeaufkommen, was zur Folge hatte, daß nahezu die Hälfte des Staatshaushalts indirekt vom Ausland finanziert wurde.2 Seit Mitte der 80er Jahre schwankte der Anteil der ausländischen Transferleistungen um $25 \%$ des Haushaltsvolumens, hat sich aber 1990/91 wieder dramatisch erhöht (vgl. Tab. 1).

1 Eine umfassende Darstellung der politischen Entwicklung des Landes und seiner wirtschaftlichen Leitlinien in den ersten zwei Jahrzehnten der Unabhängigkeit findet sich in den Beiträgen des Sammelbandes: W. Pfennig / K. Voll / H. Weber (Hg.), Entwicklungsmodell Tansania: Sozialismus in Afrika, Frankfurt / New York 1980.

2 Vgl. C. G. Kahame / T. L. Maliyamkono / S. Wells, The Challenge for Tanzania's Economy, London / Daressalam 1986, S. 158 ff. 
Als entscheidende Beschränkung bei der Suche nach einem Ausweg aus der wirtschaftlichen Zwangslage hat sich die Unfähigkeit des außenwirtschaftlich orientierten Produktionsbereichs herausgestellt, ausreichende Devisenerlöse zu erwirtschaften. ${ }^{3}$ Infolge der Abhängigkeit dieses Sektors von Zulieferimporten hat sich Tansanias Wirtschaft in einem circulus viciosus von niedriger Produktivität und unzureichenden Devisenerlösen mit entsprechend absinkendem internationalen Kreditnehmerstatus verfangen. Das Land war daher mehr und mehr vom goodwill bilateraler Wirtschaftshilfe-Geber abhängig, sah sich aber schließlich doch zu einer Kurskorrektur der Wirtschafts- und Entwicklungspolitik im Sinne der Kreditauflagen der internationalen Organisationen gezwungen. Erste Konsolidierungsschritte wurden 1982 in der widerstrebend vollzogenen Übernahme eines Structural Adjustment Programme (SAP) sichtbar, das im Staatshaushalt 1984 mit einzelnen Teilmaßnahmen einen Niederschlag fand.

Innenpolitisch hatte 1985 der Rückzug Julius Nyereres vom höchsten Staatsamt und seine Beschränkung auf den Parteivorsitz sowie die nachfolgende Wahl von Ali Hassan Mwinyi den bis dahin nur zögernd verfolgten neuen Wirtschaftskurs konsolidiert, indem der Einfluß der anti-wirtschaftsliberalen Linie im Kabinett zurückgedrängt wurde. Während die neue Exekutive nun über beträchtlichen Freiraum für wirtschaftliche Reformen verfügte, konnte sich in der Staatspartei CCM zunächst der sozialistische Flügel der Arusha-Hardliners weiter festigen. Eine Zellteilung der politischen Kräfte des Landes in Regierungsmannschaft und Parteiführung zeichnete sich ab, die lediglich durch den vermittelnden und moderierenden Einfluß des zurückgetretenen Staatspräsidenten überdeckt und überbrückt wurde. In dieser Spannungslage konnte auf dem CCM-Parteikongreß im November 1987 dann parallel zu einem radikalen Parteitagsbeschluß in einer zweiten Resolution das diametral entgegengesetzte Wirtschaftsprogramm der Regierung verabschiedet werden - ein Kuriosum, das sowohl die Zerrissenheit wie den Zwang zur Konfliktvermeidung innerhalb der Staatspartei zeigte.

Unter den Maßgaben der liberaleren Wirtschaftspolitik der Regierung Mwinyi wurden im Staatshaushalt 1986 einschneidende Konsequenzen gezogen, die eine Wiederanknüpfung der Beziehungen zu Weltwährungsfonds und Weltbank ermöglichen. ${ }^{4}$ Mit dem auf drei

3 Ebenda, S. $83 \mathrm{f}$.

4 Ein zweijähriges Stand-By-Agreement mit dem Weltwährungsfonds (IMF) (180 Mio. SDR) war 1981 eingefroren worden. Nach Einführung des SAP (1982) und des ERP (1986) stellte der IMF im Herbst 1986 einen neuen 18-monatigen Stand-By-Kredit in Höhe von 64,2 Mio. SDR bereit. Und für die dreijährige Laufzeit des ERP wurde mit WB, IMF und einer Kombination bilateraler Geber ein Kreditzufluß von jährlich rund \$ 800 Mio. vereinbart. Anfang 1990 gab der IMF nach zähen Verhandlungen die längere Zeit zurückgehaltene dritte Tranche ( $\$ 89$ Mio) des Strukturanpassungskredits frei. Die tansanische Regierung hatte hinsichtlich Währungskorrekturen und Subventionskürzungen eingelenkt. Verhandlungen über weitere Strukturanpassungsleistungen haben sich jedoch seit 1990 zunehmend aussichtslos gestaltet. 
Jahre (bis Juni 1989) berechneten Economic Recovery Programme (ERP) und dem Folgeprogramm ab 1990 (ERP II) hat die tansanische Regierung eine grundsätzliche Kehrtwende in ihrer bisher erfolglosen Wirtschaftspolitik vollzogen, die allerdings noch kaum spürbare Auswirkungen zeitigen konnte. Wesentliche Leitlinien der marktwirtschaftlichen Offnung sind: eine Zurücknahme der staatlichen Wirtschaftsintervention (Reprivatisierungsmaßnahmen), Freigabe der landwirtschaftlichen Erzeugerpreise, sukzessive Abwertung der Währung sowie Beschneidung der Staatsausgaben (Personalabbau im Staatsdienst, Subventionsabbau sowie andere Kürzungen).

\section{Haushaltspolitik und Steuersystem Tansanias}

Die Budgetentwicklung seit den siebziger Jahren war in diesem Zeitraum von zunächst rapide wachsenden Haushaltsdefiziten gekennzeichnet, die sich aus dem mangelhaft kontrollierten Anwachsen der laufenden Staatsausgaben sowie einem Schrumpfen der fiskalischen Einnahmen und der Steuerbasis erklären. Die Haushaltsdefizite erreichten von 1981 bis 1985 durchschnittlich eine Höhe von $11 \%$ des BIP. Ein Anzeichen allmählich realistischerer Haushaltspolitik ist es, daß seit 1982 dennoch die jährlichen Defizite allmählich von $41 \%(1981 / 82)$ auf $34 \%(1983 / 84)$ und rund $26 \%(1984 / 85)$ der Haushaltssummen verringert werden konnten. Seit 1986 verfolgte die Haushaltsplanung in systematischerer Form einen schrittweisen Abbau der Defizite, und die Etat-Ansätze haben seither ein Bemühen um Einhaltung dieser Leitlinien erkennen lassen. Diese hatten die Begrenzung der Defizite auf unter $2 \%$ des BIP zum Ziel sowie eingeschränkte Zuwachsraten auf der Ausgabenseite (bei weiterhin starker Betonung von Investitionen im Rahmen des Entwicklungsbudgets). Tatsächlich war in den Etats 1987/88 und 1988/89 eine verhältnismäßig strikte Einhaltung der Budgetvorgaben zu beobachten. Die Haushaltsdefizite lagen in diesen Jahren bei nur $0,4 \%$ bzw. $0,1 \%$ des BIP, bedingt allerdings durch ungewöhnlich hohe internationale Finanzspritzen. Schon 1989 hatte das Defizit wieder 7,1\% des BIP erreicht und konnte 1990/91 nur durch überproportional erhöhte ausländische Finanzzusagen nochmals vorübergehend reduziert werden (vgl. Tab. 1).

Uber den Zeitraum 1986 bis 1991 hin war nominal eine deutlich expansive Entwicklung der Haushaltsansätze zu beobachten. Sie beruht in erster Linie auf der Berücksichtigung spürbarer Abwertungen der Landeswährungen und auf der Einbeziehung der ausländischen Finanzierungszusagen in den Staatshaushalt. Erst seit 1987 spielen dabei auch Projektionen eines höheren Steueraufkommens eine Rolle, in erster Linie aus den Verkaufssteuem. Die seit einigen Jahren häufigen Abwertungen und die hohe Preisinflation machen allgemein 
die Einschätzungen der realen Ausgaben- und Einnahmenentwicklung äußerst kompliziert und undurchsichtig. 5

Die Entwicklung des Staatshaushalts ist Audruck einer langfristig entstandenen Verzerrung des gesamten Produktionssystems, die nicht nur zu Devisenengpässen und Zahlungsbilanzdefiziten geführt hat, sondern auch zu stetig verschlechterten Haushaltsbilanzen, mit der Wirkung, daß Budgetdefizite durch Bankkreditaufnahmen im Inland erneuert werden mußten. Auf der Einnahmeseite verbirgt sich hinter dieser Entwicklung ein Zurückbleiben des Steueraufkommens hinter dem Ausgabenbedarf des Staates.

Tabelle 1: Tansania: Staatshaushalt und ausländische Transferleistungen 1986 - 1991 (in Mio. TSh)

\begin{tabular}{|c|c|c|c|c|c|c|}
\hline & $1986 / 87$ & $1987 / 88$ & $1988 / 89$ & $1989 / 90$ & $1990 / 91^{*}$ & $1991 / 92 *$ \\
\hline Einnahmen & 31.102 & 59.995 & 90.201 & 98.513 & 117.134 & \\
\hline \multicolumn{7}{|l|}{ Ausgaben: } \\
\hline Verwaltungsetat & 42.699 & 60.552 & 90.272 & 118.552 & 160.000 & 186.785 \\
\hline $\begin{array}{l}\text { Entwicklungs- } \\
\text { budget }\end{array}$ & 15.860 & 17.255 & 28.400 & 22.696 & 46.000 & 41.188 \\
\hline Deckungslücke & $\begin{array}{l}27.457 \\
=46,9 \%\end{array}$ & $\begin{array}{l}17.812 \\
=22,9 \%\end{array}$ & $\begin{array}{l}28.471 \\
=23,9 \%\end{array}$ & $\begin{array}{l}42.735 \\
=30,2 \%\end{array}$ & $\begin{array}{l}88.866 \\
=43,1 \%\end{array}$ & \\
\hline
\end{tabular}

ausländische

$\begin{array}{llllll}\text { Transfer- } & 15.891 & 17.255 & 28.244 & 11.496 & 85.653\end{array}$

leistungen

$\begin{array}{llllll}\text { restliches } & 41.596 & 557 & 227 & 31.239 & 3.213\end{array}$

Haushaltsdefizit $=19,8 \%=7,2 \%=1,9 \%=22,1 \%=1,5 \%$

*) vorläufiger Haushaltsplan

Quelle: Tansania, Ministry of Finance: Financial Statements and Revenue Estimates, Daressalam 1988 und 1990

5 Die Haushaltsstatistiken der letzten Jahre sind überdies von außercrdentlichen statistischen Unsicherheiten und Abweichungen gekennzeichnet. Die Angaben divergier'n je nach Quellenlage und nach Zeitpunkt tansanischer statistischer Verlautbarungen. So sind zwischen den Angaben in den budget speeches, den "approved estimates", den "provisional actual expenditures" und statistischen Angaben ausländischer Sekundärquellen (Economic Intelligence Unit, (London) Tanzania Profile 1988/89 sowie Statistik des Auslands: Länderbericht Tansania 1987, Wiesbaden 1987) kaum annäherungsweise Übereinstimmungen zu finden. Bei der hier vorgenommenen Betrachtung stützt sich der Autor auf die letzten vorliegenden offiziellen Zusammenstellungen des tansanischen Finanzministeriums: Tanzania, Financial Statements and Revenue Estimates, vol. 1, Daressalam. Juli 1988 und 1991. 
Unter dem bestehenden Steuersystem konnte die Finanzverwaltung zwar lineare Erhöhungen des Steueraufkommens erzielen. Dieses stagniert jedoch seit langem bei ca. $19 \%$ des BIP. Von 1979 bis 1987 stieg das BIP Tansanias nominal um durchschnittlich 22,6 \% i.J., das Steueraufkommen zugleich um 23,7 \%. Dies zeigt eine ungewöhnliche Inelastizität des tansanischen Steuersystems mit einem auch im afrikanischen Vergleich geringen Auftriebsfaktor ("buoyancy") von 1,05\%;6 dabei ist überdies zu bedenken, daß auch diese Anpassung nur durch periodische Steuersatzerhöhungen, nicht aber durch die überproportionale Wirkung des Auftriebs oder verbesserte steuerliche Erfassung erzielt worden ist. Nach Einschätzung von Vertretern des internationalen Bankensystems gilt denn auch das gesamte fiskalische Einnahmensystem des Landes als "zusammengebrochen"; Steuern und Abgaben reflektieren nicht mehr den Anstieg der wirtschafltichen Aktivität.7

Im Jahr 1974 waren die zuvor bestehenden beiden individuellen Einkommensteuern zu einer einzigen zusammengefügt worden, die alle Einkommensgruppen jenseits einer Freigrenze von mtl. TSh 170 (für Stadtbewohner) bzw. 325 (für die Landbevölkerung) erfaßte, keine Abzüge i.S. des Lohnsteuerjahresausgleichs vorsah und von einer starken Progression (bis 1988: von $12,5 \%$ bis $77,5 \%$ ) gekennzeichnet war.

Da die 1969 eingeführte Verkaufssteuer die ländliche Bevölkerung überproportional belastete, wurde die städtische Bevölkerung zu Beginn der siebziger Jahre durch das Steuersystem bevorzugt. Eine spätere Reduzierung anderer ländlicher Abgaben hat diese Situation nachträglich teilweise korrigiert.

Im internationalen Vergleich zeichnet sich Tansania durch eine ungewöhnlich hohe Steuerlastquote aus und durch extrem hohe Steuersätze. Sowohl die Einkommensteuer wie die Umsatzsteuer, der Einfuhrzoll (25 - 120\%), Stempelsteuer und "estate duty" weisen so hohe Sätze und zugleich extrem niedrige Freigrenzen auf, daß sie - wenn effizient angewandt - die Haushaltsprobleme des Landes beheben müßten. Tatsächlich aber wird die Erhebung von Steuem und Gebühren nur partiell angewandt und selektiv durchgesetzt. Einerseits bestehen zahlreiche gewichtige Ausnahmeregelungen, vor allem aber wird andererseits weder der landwirtschaftliche Subsistenzsektor noch der wachsende städtische informelle Sektor in die Besteuerung einbezogen. Zur Reduzierung der Besteuerungsbasis haben auch zwei weitere wesentliche Aspekte beigetragen: Die Regierungspolitik bezüglich des Grund- und Bodeneigentums hat Grundsteuern praktisch hinfällig gemacht; und im Zollbereich ist schließlich aufgrund jahrelanger Einfuhrbeschränkungen bei der Verfolgung

6 Vergleichende Darstellungen der Steuersysteme einiger afrikanischer Länder und ihrer Verwaltung finden sich in: $U$. Fanger / F. Bolay / J. Deckers / R. Koppe, Steuerverwaltung und dienstliche Aus- und Fortbildung in Afrika - Eine Bedarfsanalyse der Länder Obervolta, Elfenbeinküste, Botswana und Kenia, Berlin 1980 (DSE-Zentralstelle für Öffentliche Verwaltung, DOK 1088 A).

7 Interviewaussagen, Vertreter der WB-Gruppe, Daressalam, Dezember 1988. 
des Import-Substitutionsmodells ein wesentlicher Teil der Besteuerungsbasis verlorengegangen; seit der schrittweisen Liberalisierung des Außenhandels zeichnet sich hier ganz allmählich eine Umkehrentwicklung ab.

Tabelle 2: Tansania: Haushalt der Zentralregierung - Aufgliederung der Einnahmen (in Mio. TSh)

$1984 / 85^{*} \quad 1985 / 86^{*} \quad 1986 / 87^{*} \quad 1987 / 88^{+}$

\section{Steuereinnahmen}

Personal + Income Tax

3.176

4.238

7.160

Sales Tax

8.288

9.821

16.620

27.193

Import Duties

735

1.491

4.042

6.483

Export Duties

10

9

0,8

andere Steuern++

540

627

1.467

1.988

Nichtsteuerliche Einnahmen

Dividends and transfers

from parastatals

503

965

826

andere Finnahmen

1.342

4.294

9.690

(Gebühren, Vergütungen,

Verkauf serlöse, Straf-

zahlungen und ausländische

Haushaltszuschüsse)

Quelle: * Govt. Printer: Economic Survey, Daressalam 1987.

+ Ministry of Finance: Financial Statements and Revenue Estimates, Daressalam 1988. Die Angaben umf assen nur Festland-Tansania, also ohne Sansibar.

++ Darin enthalten insbesondere Motor Vehicle Taxes, Production Tax, Property Taxes and Licences.

\section{Die Strukturen der Finanzverwaltung}

In ihrem Aufbau geht die Steuer- und Zollverwaltung auf eine schrittweise Neugliederung zurück, die zwichen 1973 und 1984 vollzogen worden ist. Maßgebend waren hierfür einerseits die gesetzlichen Grundlagen für die Errichtung einer umfassenden Steuerbehörde (Income Tax Act 1973), zum anderen die durch die Auflösung der Ostafrikanischen 
Gemeinschaft ${ }^{8}$ bedingte Schaffung einer eigenen tansanischen Zollverwaltung. Den neuen politischen Gegebenheiten entsprechend mußten anstelle der in Nairobi angesiedelten integrierten Zollverwaltung der drei ostafrikanischen Länder und der gemeinschaftlichen Oberbehörde der Steuerverwaltungen eigenstaatliche Behörden errichtet werden, deren Aufbauphase (insbesondere die Personalrekrutierung) sich bis 1984 hinzog.

Hatte das Finanzministerium in den ersten Jahren nach der Unabhängigkeit Tansanias als "Department of Financial Affairs" und nach seiner Aufteilung 1980 eine relativ unbedeutende Kabinettsposition eingenommen, so ist dem Ressort durch die Wiedervereinigung (1986) der Finanzverwaltung mit dem stets stark politisch beeinflußten Ressortbereich für Wirtschaft und Entwicklungsplanung unter der gegenwärtigen Regierung und mit Finanzminister Cleopa Msuya und seinem seit März 1990 amtierenden Nachfolger Stephen Kibona eine herausragende politische Stellung zugewachsen. Steuer- und Zollverwaltung sind seither Teil dieses im Lauf der Jahre mehrfach in seinem Zuständigkeitsbereich veränderten Ministeriums für Finanzen, Wirtschaft und Planung, während das Schatzamt (Treasury) seit 1961 Teil des Amtes des Prime Minister geblieben ist.

In der gegenwärtigen Struktur der Ministerialverwaltung stellen Steuer- und Zollverwaltung je eine eigene Hauptabteilung (department) dar, neben dem Department of Financial Affairs, dem Department of Economic Affairs und dem Department of Planning, von denen sie räumlich getrennt sind. Die Vereinigung der Steuerbehörde mit der Zolladministration, die zuvor in der Hafenabfertigung untergebracht war, konnte erst 1984 vollzogen werden.

Offensichtliche Engpässe in der organisatorisch schlecht bewältigten Überleitungsphase veranlaßten das Finanzministerium 1978 zur Einholung eines Gutachtens vom Internationalen Währungsfonds (dessen Empfehlungen verworfen wurden) und 1981 zur Einsetzung einer Task Force, deren Lösungsansätze ebenfalls nicht als praktikabel angesehen wurden. Seit 1983 war der Hauptabteilung Zoll ein mehrjähriges Beratungsprojekt der UNDP beigegeben; dieses entwickelte ein eigenes organisatorisches Reformmodell, das großenteils akzeptiert und umgesetzt werden konnte. 9

8 Obwohl die ostafrikanische Gemeinschaft bereits 1977 aufgelöst worden war, hatte die gemeinsame Zollverwaltung noch während einiger Übergangsjahre Bestand. Die Verkaufssteuer (Sales Tax) war dagegen außerhalb der Gemeinschaftszuständigkeit geblieben und wurde vom Tanzanian Intemal Revenue Department zusammen mit mehreren anderen inländischen Einzelsteuem verwaltet.

9 Die UNDP-Vorschläge haben ihren Niederschlag gefunden in einem vertraulichen Bericht des Finanzministeriums: Ministry of Finance, Economic Affairs and Planning: Report on Reorganisation of Department of Customs and Sales Tax, Daressalam, Juli 1986. Die früheren Vorschläge des IMF sahen vor allem eine Zusammenlegung des Revenue Department mit dem Income Tax Department vor. Da dies die verwaltungsmäßige Verbindung disparater Steuerarten bedeutet hätte, wurden die Vorschläge nach Kabinettsberatung zu den Akten gelegt. 
Abgesehen von der Finanzverwaltung der Gebietskörperschaften (Local Government Financial Administration) werden Steuer- und Zollaufgaben zum einen durch das Income Tax Department (Hauptabteilung Einkommensteuer, ITD), zum anderen durch das Customs and Sales Tax Department (Hauptabteilung Zoll, CSTD) wahrgenommen. Beide Abteilungen stehen unter der Leitung von Commissioners (Commissioner for Income Tax and Commissioner for Customs and Sales Tax); sie sind dem Vizeminister für Financial Affairs bzw. dem diesen beratenden Deputy Secretary unterstellt und verantwortlich.

Die Zentrale Verwaltung des Income Tax Department gliedert sich in fünf Unterabteilungen (divisions), die von Assistant Commissioners geführt werden. Es handelt sich dabei um Unterabteilungen für

- Körperschaftsteuer (Corporate Taxation)

- Individuelle Einkommensteuer (Non-Corporate Taxation)

- Steuerprüfung und -fahndung (Audit and Investigation)

- Steuererhebung und -beitreibung (Collection and Compliance)

- Personalverwaltung und Arbeitsvorbereitung (Manpower Development and Administration, Tax Management).

Außerhalb der Zentralverwaltung bestehen regionale Finanzämter (Regional Headquarters) in den 21 Regionen des Landes (einschließlich Sansibar), die jeweils für durchschnittlich fünf Verwaltungsbezirke zuständig sind. Sie werden in der Regel von einem District Officer geleitet und verfügen jeweils über einen Personalstab von zwischen 12 und 16 Steuerbeamten. 10

\section{Der tansanische öffentliche Dienst und die Personalkapazitäten der Steuerverwaltung}

Nach der Unabhängigkeit Tansanias wurde das aus der britischen Verwaltungstradition übernommene System des Civil Service zunächst bis 1967 beibehalten, jedoch durch den Civil Service Act von 1962 mit neuen gesetzlichen Grundlagen versehen, zu denen die Schaffung der Civil Service Commission gehörte.11 Diese teilte sich die Aufgaben der Rekrutierung öffentlicher Bediensteter mit fünf weiteren Behörden, deren Funktionen und Einflußbereiche sich teilweise überlappten. Entscheidend für die Besetzung der Spitzenpositionen des öffentlichen Dienstes wurde die Central Establishment Division im Präsidialamt, die für die Auswahl und Emennung aller Dienstgruppen in Gehaltskategorien eines

10 Die geringste Besetzung findet sich im regionalen Finanzamt Lindi mit 12, die stärkste Besetzung in Sansibar mit 16 Steuerbeamten.

11 Eine detaillierte Darstellung des zentralen und lokalen Verwaltungssystems vor und nach der Unabhängigkeit Tansanias bringt $K$. W. von Sperber, Public Administration in Tanzania, München 1970, insb. S. 53-65. 
Jahresverdienstes von mehr als 37.800 TSh zuständig war. Diese Entscheidungsposition für politisch relevante Postenbesetzungen ist bis in die Gegenwart dem Präsidialamt vorbehalten geblieben.

Wesentliche strukturelle Veränderungen fanden 1972 und 1974 sowie schließlich 1980 statt. Sie waren gekennzeichnet durch die Konsequenzen der Regionalisierungspolitik für den öffentlichen Dienst 12 und durch Schaffung des Ministry of Human Resources and Manpower Development (1974), das direkt dem Präsidialamt nachgeordnet wurde und dessen Principal Secretary zugleich die Leitung des gesamten öffentlichen Dienstes innehatte.

Da die neuen Regional-Verwaltungsdienste dem Amt des Premierministers unterstanden und dieses dadurch politisch gestärkt worden war, ergab sich nunmehr eine parallele Zuständigkeit für zwei Sektoren des öffentlichen Dienstes. Es entstanden Reibungsverluste und Probleme, die 1980 zugunsten einer Übertragung der gesamten Zuständigkeit für alle Staatsbediensteten auf das Amt des Premierministers gelöst wurden. Seither ist der Principal Secretary im Prime Minister's Office oberster Dienstherr aller Staatsbediensteten. Die Verteilung der Zuständigkeiten für Emennungen in sachbestimmten Dienstgruppen bleibt jedoch bestehen.

Eine der auffallendsten Erscheinungen in der Entwicklung der tansanischen Beamtenschaft seit den 60er Jahren ist die ungestüme Ausweitung des Personalbestandes (1967: insg. 65.708 Bedienstete; 1980: 295.352; 1988: 302.632, davon 142.166 im zentralen Staatsdienst und 160.466 in den Kommunalverwaltungen). Dieses Anwachsen der Staatsbürokratie ging in mehreren zeitlichen Schüben vor sich. Die Tatsache, daß sie sich überproportional auf die untersten Dienstgruppen erstreckt hat, während sich zugleich in den mittleren und gehobenen Diensträngen hohe Zahlen unbesetzter Stellen (6.065 Vakanzen aus insgesamt 16.805 Posten) ergeben, zeigt einerseits die sozialpolitische Zielrichtung der Rekrutierungspolitik, andererseits aber auch die Schwierigkeit, qualifizierte Absolventen für mittlere und gehobene Aufgaben im öffentlichen Dienst unter den vorgegebenen Besoldungsrichtlinien heranzuziehen. 13 Die hier für den gesamten Verwaltungsdienst angerissene Pro-

12 1972: Schaffung von zunächst 17, später 21 Regionen, die jeweils in den Rang von Ministerien erhoben wurden; zugleich Auflösung der traditionellen Lokalverwaltungen. In der Praxis bedeutete die Schaffung der Regionen, daß die Regional Development Directors die Funktion von "Emennungsbehörden" erhalten haben, so daß sie - wie die Principal Secretaries eines Ministeriums - Personaleinstellungen für operative Dienstgruppen (bis zum Jahresverdienst von 10.260 TSh) eigenständig vomehmen. Zur "Regionalisierung" vgl. im übrigen D.-C. Martin, Tanzanie - L 'invention d'une culture politique, Paris 1988, S. $94 \mathrm{ff}$.

13 Vgl. R. S. Mukandala, Bureaucracy and Socialism in Tanzania: The Case of the Civil Service, in: The African Review, Bd. 10 (1983) 2, S. 1-20 sowie ders., Trends in Civil Service and Income in Tanzania, 1967-82, in: Canadian Joumal of African Studies, 17 (1983) 2, S. 253-263. 
blematik des Qualifikations- und Stellenkegels trifft speziell auch auf die Finanzverwaltung zu, wie im einzelnen noch zu erläutem sein wird. Für die Beschäftigten der zentralen Ministerien gilt ein Klassifikationsschema nach 14 Dienstklassen, von MS 1 (MS = Ministerial Staff) als niedrigster bis MS 14 als höchster Kategorie. Innerhalb der Klassen wird differenziert nach Grad III - I, wobei I die höchste Stufe bezeichnet. Die charakteristischen Positions- und Aufgabenbezeichnungen in der Finanzverwaltung lassen sich - wie in der folgenden tabellarischen Aufstellung gezeigt (Tab.3) - in dieses Klassifikationsschema einordnen. Dabei soll versucht werden, auch eine annähernde Zuordnung von BruttoMonatsgehältern zu vermerken. Wie ersichtlich, ist das Besoldungsniveau außerordentlich niedrig, nicht nur in den unteren und mittleren Dienstgruppen. Dies erklärt sich aus der Finanzlage des Landes, die keine regelmäßigen Gehaltserhöhungen zugelassen hat, entspricht aber auch der seit der Unabhängigkeit verfolgten Orientierung am "Fabianischen Sozialismus", mit dem tendenziell eine Annäherung und Einebnung der Extreme in der Lohn- und Gehaltsskala angestrebt und verfolgt wurde.

Tabelle 3: Dienstgruppen-Klassifikation für das Ministry of Finance / Steuer- und Zollverwaltung (Stand: Mitte 1988)

MS 1 - 4 Untere Dienstgruppen (clerical staff, tax collecting clercs, tax collecting assistants; accounting assistants and accountants ab MS 3: tax collection officers mit mehr als dreijähriger Berufserfahrung; MS 4: Officers mit langjähriger $\mathrm{Be}$ rufserfahrung)

MS 5 - 7 Mittlere Dienstgruppe (Aufsichtsfunktionen und erhöhte Verantwortung; eigenständige Bearbeitung der Tarifklassifikation. Financial Management Officer III, Senior Collector, Sektionsleiter, Chef des Regionalamtes u.ä.)

MS 8 - 11 Höhere Dienstgruppe mit ManagementAufgaben (Senior Financial Management Officer, Principal Collector ...)
Gesetzlicher Mindestlohn: 1600 Sh (Hauptstadt) oder 1200 Sh (Provinz) +600 Sh Hauptstadtzuschlag

2000 bis $3000 \mathrm{Sh}$;

Endgehalt: $5000 \mathrm{Sh}$

2500 bis $3500 \mathrm{Sh}$ 
Im höheren Dienst mit Sonderaufgaben:

MS 12 Assistent des Hauptabteilungsleiters (Assistant Commissioner)

MS 13 Stellvertretender Hauptabteilungsleiter (Deputy Commissioner)

MS 14 Hauptabteilungsleiter (Commissioner)
4000 bis $5000 \mathrm{Sh}$

dito zzgl. Wohnungszulage (4000 bis $5000 \mathrm{Sh}$ )

dito zzgl. Wohnungszulage $6000 \mathrm{Sh}$

dito zzgl. Wohnungszulage und Dienst-Pkw

(Vergütung monatlich in $\mathrm{Sh})^{14}$

Die Gehälter bewegen sich - annähernd entsprechend der Kaufkraftrelation der Währung umgerechnet (vgl. Anm. 14) - zwischen monatlich DM 12 für die unterste Dienststufe (bei Einsatz in der Provinz) und DM 60 für die höchste Dienststufe (Commissioner, etwa entsprechend dem Rang eines Unterstaatssekretärs im Finanzministerium). Zu berücksichtigen ist, daß die Arbeitszeit in der Steuer- und Zollverwaltung wie in allen zentralen Behörden auf fünf Stunden am Vormittag beschränkt ist, allerdings mit Ausnahme des Spitzenpersonals, das überdies häufig zusätzlich an Wochenenden Dienst tut. Die Staatsverwaltung vertraut somit grundsätzlich darauf, daß der Lebensunterhalt durch private nebenberufliche Tätigkeit abgesichert wird. Dennoch bringt es die - auch im Vergleichsmaßstab anderer Länder der Dritten Welt - extrem unzureichende Besoldung mit sich, da Phänomene einer Versorgungskorruption zwangsläufig entstehen und verfestigt werden.

Entsprechend der überragenden Bedeutung der Umsatzsteuem innerhalb der gesamten Staatseinnahmen weisen die beiden Hauptabteilungen der Finanzverwaltung eine beträchtlich unterchiedliche Personalbesetzung auf. In der Einkommensteuer-Abteilung (ITD) sind insgesamt rd. 1.500 Bedienstete tätig, in der Abteilung Zoll und Umsatzsteuer (CST) rd. 2.950 Bedienstete.15 Um einen annähemden Überblick über die Aufgliederung des Personalbestandes, getrennt nach den beiden Hauptabteilungen, zu erhalten, wird hier auf ältere statistische Angaben (Anfang 1987) zurückgegriffen. Seither sind Personalaufstockungen,

14 Angesichts der hochgradigen Preisinflation war mit Wirkung zum 1.1.1989 erstmals seit längerer Zeit für den öffentlichen Dienst eine Besoldungserhöhung verfügt worden. Danach beträgt die Zulage für die untere Dienstgruppe $20 \%$, ab der mittleren Dienstgruppe $10 \%$. Für die Umrechnung in DM galt (1989) der offizielle Kurs von $1 \mathrm{DM}=67 \mathrm{TSh}$. Wirklichkeitsnäher im Hinblick auf die Kaufkraft war die Zugrundelegung eines mittleren Freikurses von $1 \mathrm{DM}=100 \mathrm{TSh}$.

15 Ergebnisse der Einsichtnahme in Unterlagen der Personalverwaltung beider Abteilungen des Finanzministeriums, Daressalam, 1989. 
insbesondere in den unteren Dienstgruppen, erfolgt. Im ITD liegt der Anteil der Fachbeamten höherer Dienstgruppen (officer cadre) bei knapp unter 30 \% des Gesamtpersonals. Der Personalstab dieser Unterabteilung umfaßte 1988/89 161 Assessors, denen 635 Sachbearbeiter und 261 Hilfskräfte zugeordnet waren.

Income Tax Department (1987, nur Zentralverwaltung)

Leitungspersonal (Commissioner und Assistant Commissioners, MS 14/12) 5

$\begin{array}{ll}\text { Principal Assessors } & 17\end{array}$

Senior Assessors $\quad 22$

$\begin{array}{ll}\text { Assessors } & 170\end{array}$

Tax Management Officers (clerical level) $\quad 635$

Sekretariatspersonal und untere Verwaltungsdienste $\frac{261}{1.110}$

Der Hauptabteilungsleiter (Commissioner of Income Tax, CIT) wird unmittelbar vom Staatspräsidenten emannt. Für diese Position ist keine konkrete Laufbahndefinition vorgegeben, die Emennung muß allerdings aus dem höheren Dienst des Finanzministeriumis erfolgen. Dem CIT sind für die Zuständigkeitsbereiche Körperschaftsteuer, individuelle Einkommensteuer, Steuerprüfung und -fahndung sowie Steuererhebung vier Assistant Commissioners unmittelbar zugeordnet. Der Bereich Personalverwaltung/Arbeitsvorbereitung wird von einem der Principal Assessors geleitet.

Aufgrund der herausragenden wirtschaftlichen und kommerziellen Bedeutung der Hafenmetropole und Hauptstadt Daressalam nimmt dieser Bezirk innerhalb der Gebietsgliederung der Finanzverwaltung in Umfang und Personalausstattung eine Sonderstellung ein. 16 Der Hauptstadtbezirk ist als Sonderregion aus der Finanzverwaltung des umliegenden Amtsbezirks ausgegliedert. Die Dienststelle verfügt über eine wesentlich umfangreichere Personalausstattung als die anderen Regionalämter, gilt aber in Anbetracht des überproportionalen Aufgabenvolumens als kritisch unterbesetzt. Hier ist ein gesonderer Regional Principal Assessor zuständig, der unmittelbar dem CIT gegenüber verantwortlich ist. In den zwei Unterabteilungen (technical divisions) des Regionalfinanzamtes, zuständig einerseits

16 Der Steuerbezirk Daressalam erwirtschaftet i.d.R. zwischen $70 \%$ und $75 \%$ des gesamten Steueraufkommens des Landes. Für 1987 ergab sich ein Anteil von 54 Mrd. TSh von insg. 76 Mrd. TSh. Im Zeitraum Juni - November 1988 erzielte das Regional-Finanzamt mit 26,6 Mio. TSh $92 \%$ der gesamten Steuereingänge. Der Grund liegt darin, daß sich in der Zentralregion i.d.R. ein zeitlicher Vorsprung beim Steuereinzug gegenüber den anderen Landesteilen erzielen läßt. Dies ist eine Folge der überproportionalen Konzentration körperschaftsteuerpflichtiger juristischer Personen und dem Lohnsteuer-Vorabzug angeschlossener Untemehmen in der Hauptstadtregion. Bezogen auf das Fiskaljahr 1987/88 erzielte dieses Finanzamt ein Aufkommen von rd. 380 Mio. Tsh. und übertraf damit die Zielvorgabe (311 Mio TSh) um ca. $22 \%$; Quelle: Interviewaussagen und Akteneinsicht, Daressalam Regional Office. 
für Körperschaftsteuer, andererseits für die individuelle Einkommensteuer, sind unter jeweils einem Senior Tax Assessor insgesamt 55 Steuerbeamte tätig, davon 38 Assessors. Zusammen mit 110 übrigen Bediensteten macht der Personalstab des Regionalfinanzamtes Daressalam etwa $15 \%$ des Personalbestandes der Hauptabteilung aus. Allerdings sind darüber hinaus weitere ca. 300 Personen in der Zentralbehörde teilweise mit Steuervorgängen des Hauptstadtbereiches befaßt.

Die Steuerverwaltung stützt sich landesweit auf Regional-Finanzämter in jeder der 21 Regionen, 17 mit zwischen 12 und 16 Bediensteten, jeweils unter der Leitung eines Regional Head Officer, in der Regel einem Principal Assessor, in den nach Steueraufkommen weniger bedeutenden Ämtern auch Senior Assessors (meist Gehaltsgruppe MS 6). Der Hauptpersonalstab umfaßt in den meisten Fällen einen Financial Management Assistant (MS 4) und zwei Bedienstete der gleichen Ausbildung nach Gehaltsgruppe MS 3.18

Nach Aufstockung des Personalbestandes im Jahre 1990 ist die Steuerverwaltung in 88 der insgesamt 114 (Landkreis-) Bezirke mit zumindest einem Inspektor (Assessor) vertreten, der jedoch kein eigenes Bezirksbüro führt, sondern innerhalb des Regional-Finanzamtes für einen Bezirk zuständig ist und diesen bereist.

Customs and Sales Tax Department (1987, nur Zentralverwaltung)

Leitungspersonal (Commissioner und 3 Assistant Commissioners, MS 14/12) 4

Senior Finance Management Officials (Principal bzw. Senior Auditors, MS 10) 6

$\begin{array}{lr}\text { Finance Management Officials (Middle Level, MS 5-9) } & 170\end{array}$

Finance Management Officials (Middle Level, MS 4) 180

Auditors MS 3 ca. 1.100

Auditors MS 2 ca. 1.460

Sekretariats-/Bürohilfspersonal, Zollageraufsicht etc. $\quad 2.530$

Für die Position des Hauptabteilungsleiters (Commissioner of Customs and Sales Tax) gilt entsprechend das über den gleichwertigen Rang der Hauptabteilung ITD Gesagte. Dem

17 Die Struktur der an die Zentralverwaltung angebundenen 21 Regionalämter wird lediglich in bezug auf die Steuerfahndung durchbrochen, indem als zusätzliche Ebene jeweils 3 - 4 Zonen zusammengefaßt werden. Der Sitz der Fahndungsbehörde ist jeweils einem der Regionalämter beigegeben. Für den gesamten Südostteil des Landes (4 Regionen der südlichen Küste und des Landesinneren) fungiert ein Teil der Zentralverwaltung im ITD als Zonal Office.

18 Die Angestellten der mittleren Dienstgruppe (MS 4-9) beaufsichtigen jeweils etwa 15 Angestellte, als Chef einer Sektion haben sie 3-5 Bedienstete der Gehaltsgruppe MS 4 unter sich. Die Gehaltsgruppe MS 3 stellt für sich den größten Personalanteil innerhalb der Zoll- und Umsatzsteuerabteilung dar; sie rekrutiert sich durch Aufstieg aus der Gruppe MS 2. Zur Regelbeförderung gelangen jeweils ca. $10 \%$ der unteren Einstiegsgruppe nach 3 - 4 Jahren praktischer Erfahrung und Leistungsnachweis. 
Commissioner sind unmittelbar drei Assistant Commissioners und ein Principal Finance Management Official mit konkreten übergreifenden Aufgabenbereichen zugeordnet (Administration and Accounts; Customs Operations; Sales Tax and other taxes; Investigation and Audit) sowie zwei der Principal Auditors (für Policy and Research und Legal and International Affairs). Auf regionaler Ebene ist das CST mit eigenen Ämtern an den Grenzen und in den Hafenstädten vertreten, im übrigen nur in Form von Resident Sales Tax Officers in den Regional-Finanzämtern. Ihnen stehen in der Regel ein Revenue Inspector und ein Investigation Officer zur Verfügung sowie Revenue Agents (Collection and Accounting) für die einzelnen Landkreis-Bezirke.

\section{Probleme des Personalbestandes und der Rekrutierung}

Eines der Hauptprobleme der Finanzverwaltung ist die - in quantitativer, aber auch qualitativer Hinsicht - unzureichende Personalausstattung. Sie stellt ein entscheidendes Hindernis für die Steuer- und Zollverwaltung dar, den Zielvorgaben für eine Effizienz- und Ertragsverbesserung der steuerlichen Erfassung nachzukommen.

Beide Hauptabteilungen für Steuer- bzw. Zollverwaltung weisen (mit 1.500 bzw. 2.950 Stellen) eine geringere Personalbesetzung auf, als ihnen in den Haushaltsansätzen zugewiesen ist. Die Diskrepanz zwischen den ursprünglich vom Ministry of Manpower Development zuerkannten "authorized posts" und dem tatsächlich genehmigten Stellenplan ișt besonders auffällig bei der Hauptabteilung Zoll/Umsatzsteuer (rd. $3.000 \mathrm{im}$ Vergleich zu 4.169 Stellen); bei der Hauptabteilung Einkommensteuer beträgt die Differenz $1.500 \mathrm{zu}$ rd. 1.900. Dieses Mißverhältnis hat zwei Erklärungshintergründe, die wiederum beide ihren Ursprung in den Haushaltsengpässen und der mangelnden Finanzausstattung der Staatsverwaltung haben:

- Bis einschließlich 1987 galt für den öffentlichen Dienst allgemein eine langjährige Einstellungssperre ("recruitment embargo"), die für die Finanzverwaltung erst für 1988 durch Beschluß einer speziell einberufenen Sonderkommission und durch Ausnahmegenehmigung des Ministry of Manpower Planning aufgehoben worden ist.

- Für den Eintritt in die mittleren und höheren Laufbahngruppen stellen sich so wenige Bewerber mit entsprechenden Schul- bzw. Hochschulabschlüssen, daß über mehrere Jahre hin der natürliche Abgang (insbesondere durch Eintritt in den Ruhestand) 19 nicht ausgeglichen werden konnte.

19 Freiwillige Frühpensionierung mit 50 J., Ruhestand i.d.R. mit 55. J. 
In ihrem Zusammenwirken haben beide Faktoren zu einer spürbaren Ausdünnung im Mittelbau der Verwaltung, d.h. bei der Gruppe der spezialisierten, langjährig erprobten und mit Aufsichtsfunktionen ausgestatteten Inspektoren und Oberinspektoren geführt. Eine weitere Folge ist die außerordentlich hohe Fluktuationsrate im Finanzdienst durch Abwanderung in die Privatwirtschaft nach mehrjähriger Erfahrung im öffentlichen Dienst. Die bis 1987 eingehaltene Einstellungssperre hat die Finanzverwaltung daher ausgedünnt. Seither zeigt sich, daß zwar die Rekrutierung von Bewerbern für die Eingangsstufen der unteren Dienstgruppe allgemein unproblematisch ist, aber schon für die Beförderung in mittlere Ränge keine ausreichende Zahl qualifizierter Bewerber zur Verfügung steht. Hinzu kommt, daß der Bewerberkreis aus Oberschulabsolventen für die mittleren und höheren Dienstgruppen extrem klein ist und ein Großteil der Abiturienten sich nicht zum Eintritt in den öffentlichen Dienst entschließt. Insgesamt wird von der tansanischen Finanzverwaltung beständig auf die Dringlichkeit von Personalaufstockungen hingewiesen. Tatsächlich ist die geringe Zahl höherer Steuerbeamter mit langjähriger Diensterfahrung seit Jahren eine der größten Schwächen der Verwaltung. Die wiederholte pauschale Forderung nach ca. 900 neuen Posten beim Zoll und rd. 400 neuzuschaffenden Eingangspositionen (assessor trainees) für das ITD, mit der Argumentation, daß dies dann quasi automatisch eine wesentliche Erhöhung des staatlichen Steueraufkommens nach sich zöge, trifft nicht den Kern des Problems und würde kaum zur Problemlösung führen. Denn der Großteil des Steueraufkommens stammt nicht aus dem einkommensteuerlichen Individualbereich, in dem detaillierte, personalaufwendige Erklärungen anfallen (nur ca. $12 \%$ der Einnahmen), sondern aus dem Körperschaftste:iseraufkommen der 443 parastaatlichen und rd. 10.000 privaten Unternehmen vornehmlich im Großraum Daressalam und Arusha (ca. $55 \%$ ) und aus dem weniger personalintensiven PAYE-Lohnabzugsverfahren (rd. $25 \%$ ). Auch seitens der Finanzverwaltung wird eingeräumt, daß insbesondere im ITD administrative Effizienzverbesserungen (Reorganisation des Personaleinsatzes, Neuzuordnung von Verantwortlichkeiten, verbesserte Beaufsichtigung, Anleitung und Fortbildung der jüngeren Inspektoren) und Fortbildungsmaßnahmen einen Teil der Personallücke ausgleichen könnten.

Nach Lockerung der Einstellungssperre wurde seit 1987/88 tatsächlich wieder eine Personalaufstockung in beiden Hauptabteilungen um insgesamt rd. $10 \%$ erzielt. Die jährlichen Neueinstellungen sollen sich ab 1988 belaufen auf je 30 assessor trainees (Graduierte oder Diplomierte), 60 collection of ficials sowie 100 tax clerks im ITD und 120 custom clerks im CST. Im Zuge der Bemühungen, speziell die Personalsituation im Hinterland zu verbessem und hier eine Erfassung auch des informellen Sektors in den Landbezirken zu erreichen, wurden im Zoll-/Umsatzsteuerbereich rd. $75 \%$ der in 1988 neu Aufgenommenen auf der Regionalebene eingesetzt, ein Viertel in Daressalam. Das Ziel einer systematischen Verbesserung auf der Regional- und Distriktebene wird aber nur sehr langfristig zu erreichen sein. Da aus dem Hochschulsystem im ganzen Land jährlich nur eine kleine Zahl von Absolventen hervorgeht (1983: nur 18; 1988: 61), sind allein von der Nachfrageseite für die kommenden Jahre keine so hohen Neuzugänge für die mittleren und gehobenen Dienst- 
gruppen zu erwarten. So konnte das ITD in den letzten Jahren nicht einmal seine Quote für Ausbildungsstellen von tax collection assistants ausschöpfen, da nicht genügend Bewerber mit Grundschuldiplom zur Verfügung standen. Bei Ausschreibungen für die mittlere Dienstgruppe der Finanzverwaltung (College-Abschluß) melden sich wenige oder keine Bewerber, da es "alle vorziehen, sich um die staatlichen Wirtschaftsunternehmen und Körperschaften zu bewerben".20 Erst durch Quotenverteilung seitens des Personalausschusses im Ministry of Manpower Planning werden der Finanzverwaltung Bewerber zugewiesen.

Für 1988 und 1989 wurde erstmals ein zahlenmäßiges Gleichgewicht zwischen den Ausscheidenden und Neuzugängen in den mittleren Dienstgruppen erreicht, aber das "Altersgruppenloch" besteht weiter, und die negativen Folgeerscheinungen in qualitativer Hinsicht verstärken sich weiterhin: Es tritt ein Verlust an erfahrenen Beamten ein, die nur durch unerfahrene Neuzugänge ersetzt werden können; Schlüsselstellen werden in zunehmendem Umfang ersatzweise von niedrigrangigen Beamten wahrgenommen.

Der Personalbestand der Steuer- und Zollverwaltung weist daher bemerkenswerte quantitative Lücken insbesondere in den mittleren Dienstgruppen sowie qualitative Defizite auf. Sie sind das Ergebnis zahlreicher Einflüsse, unter denen in erster Linie zu nennen sind: das extrem niedrige Gehaltsniveau (mit der Wirkung mangelnder Attraktivität des öffentlichen Dienstes, hoher Personalfluktuation, fehlender Leistungsanreize usw.), die personelle Ausdünnung infolge mehrjähriger Einstellungssperre und die niedrigen Absolventenzahlen des tansanischen Oberschul- und insbesondere Hochschulwesens. Es muß aber betont werden, $\mathrm{da} ß$ eine allgemeine Personalaufstockung in der besonderen Situation der tansanischen Steuerverwaltung nur effizient sein kann, wenn sie mit einer durchgreifend verbesserten Erfassung der Individualbesteuerung verbunden werden soll.

\section{Verwaltungsverfahren und administrative Engpässe21}

Der Zielbereich der tansanischen Steuerverwaltung umfaßte nach dem Stand von 1989 knapp 200.000 einkommensteuerpflichtige natürliche Personen (einschl. Personengesellschaften) und rd. 32.000 körperschaftsteuerpflichtige juristische Personen.22 Unter Einbeziehung der Fälle von Lohnsteuerabzug durch rd. 5.800 Arbeitgeber nach dem "Pay as you

20 Gesprächsinformation, Income Tax Department Daressalam / Regional Office Morogoro.

21 Bei den Ausführungen dieses Abschnitts konnte sich der Verfasser dankenswerterweise vor allem auf Aussagen von Prof. Christian A. Rasenack (TU Berlin) stützen, die in einem gemeinsamen unveröffentlichten Berichtsmanuskript niedergelegt sind.

22 Die hohe Zahl der körperschaftsteuerpflichtigen Personen dürfte mit der hohen Zahl der landwirtschaftlichen Genossenschaften zu tun haben, die im Ergebnis steuerbefreit sind, aber offenkundig gleichwohl steuerlich registriert werden. Die Staatsgesellschaften erbringen insg. etwas mehr als die Hälfte des Aufkommens aus dem körperschaftsteuerlichen Teil der Einkommensteuer. 
earn"-Verfahren (PAYE), von dem 700.000 Arbeitnehmer betroffen sind, und einigen besonderen Erhebungsformen (wie Pay-roll Levy und Training Levy) ergaben sich daraus insgesamt ca. 235.000 Steuerfälle.23

Veranlagt wird anhand sog. vorläufiger Steuererklärungen, die von den Steuerpflichtigen bis Ende des dritten (im Fall der Körperschaftsteuern bis Ende des sechsten) Monats jeweils für das Vorjahr abgegeben werden sollen. Die Einkommensteuer entspricht in Umfang und Art im großen und ganzen einer in Westeuropa üblichen Steuererklärung. Da der zuständige Verwaltungsbeamte außerdem zusätzliche Informationen einholen kann, soweit Zweifel an den Angaben des Steuerpflichtigen bestehen, liegt dem äußeren Erscheinungsbild nach mithin das Modell einer Amtsveranlagung zugrunde. Die Steuer wird zeitnah erhoben, allerdings nicht unter Einsatz von später anzurechnenden Vorauszahlungen, sondern aufgrund der zu Beginn des Steuerjahres abgegebenen vorläufigen Selbsteinschätzung. Nach der Absicht des Gesetzgebers sollten die auf dieser vorläufigen Festsetzung beruhenden Zahlungen bei einer endgültigen Veranlagung verrechnet werden. In der Praxis weicht die Sachlage weitgehend von diesen Richtlinien ab, und hier scheint eine der gravierenden Erhebungsschwächen des tansanischen Verwaltungsapparates zu liegen. die Einkommensteuerabteilung wird.mit vorläufigen Erklärungen überschwemmt, die in erster Linie deshalb vorgelegt werden, weil sie die Voraussetzung für die Erteilung der Handelslizenzen sind. Ein hohes $\mathrm{Ma} ß$ an willkürlicher Selbsteinschätzung dürfte in dieses Verfahren einfließen, selbst wenn der Fall mit dem Veranlagungsbeamten besprochen oder ausgehandelt wurde. Wesentlicher ist jedoch, daß im überwiegenden Teil der Fälle keine Abschlußerklärung (retum) nachgereicht wird und die Veranlagung in der Regel aufgrund der vorläufigen Selbsteinschätzung der Steuerpflichtigen erfolgt.

Auch bei den Fällen - in der Regel größere Steuerzahler und "auffällige" Steuerakten -, in denen eine zweite, endgültige Erklärung nachgereicht wird, erscheint der Aussagegehalt oft wenig zuverlässig. Einerseits ist nämlich die Befolgung hergebrachter Grundsätze ordnungsgemäßer Buchführung nicht nur bei den natürlichen Personen außerordentlich gering entwickelt, 24 andererseits scheint auch das Erfordemis, daß bestimmte Steuerzahler ihren Erklärungen Nebenerklärungen (Bilanzen usw.) beifügen müssen, die von staatlich anerkannten Buchprüfem gegenzuzeichnen sind, ebenfalls keine wirklichkeitsnäheren Steuererklärungen zu zeitigen. Das Land verfügt insgesamt nicht über die erforderliche Zahl ausgebildeter Buchhalter, Buchprüfer und Steuerberater, geschweige denn über die entsprechend ausgebildeten Veranlagungsbeamten. Hinter entsprechenden Erklärungen verbirgt sich im allgemeinen daher wohl wenig mehr als eine in der Regel für den Steuerpflichtigen zu günstige grobe Schätzung. Im tansanischen Steuerwesen ist auf diese Weise

23 Interviewaussagen, Finanzministerium Tansania, Income Tax Department.

24 Selbst von den staatlichen Kapitalgesellschaften wurde berichtet, daß sie keine verläßliche Buchführung besitzen. 
aus der beabsichtigten Amtsveranlagung eine Selbsteinschätzung der Steuerbürger geworden, wodurch massive Einnahmeausfälle entstehen. Zu durchbrechen wäre die eingefahrene administrative Praxis nur durch ersatzlose Abschaffung der vorläufigen Erklärung und die Einführung von Vorauszahlungen auf der Basis der vor jährigen Amtsveranlagung. Für eine solche Reform fehlen jedoch zunächst noch die Voraussetzungen. Hilfsweise müßten schrittweise Verbesserungen dahingehend eingeführt werden, daß Verfahren der Schätzung (standard assessment) systematisch eingesetzt werden. Dafür wiederum sind zunächst auch die Methoden des inneren und äußeren Betriebsvergleichs und deren Voraussetzungen (Buchführung, Inventur, Kostenrechnung) zu vermitteln. Erforderlich ist jedoch auch die Vermittlung der damit einhergehenden Verfahrensweisen (Nachschau, Erzwingung der Mitwirkungsverpflichtung, Androhung steuerstrafrechtlicher Maßnahmen) und die Propagierung einer rationaleren Form der Aktenführung, die Gruppen von Steuerpflichtigen enger an konkrete Veranlagungsbeamte bindet. hier liegt eine der sofort ins Auge fallenden administrativen Schwachstellen; die Steuerakten werden zentral gelagert, verstauben infolgedessen in dicht bepackten riesigen Regalen oder werden in ungeordneten Haufen gestapelt. Obwohl Listen geführt werden, ist offensichtlich unter diesen Bedingungen eine echte Aktenkontrolle nicht einmal annäherungsweise möglich. Weitgehend dem Zufall überlassen bleibt daher die Verfolgung unabgeklärter Steuerfälle; die Querkontrolle verschiedener Steuerarten und die systematische Auswertung offengelegter Steuerfälle durch Ausschreiben von Kontrollmitteilungen unterbleibt. Im übrigen ist die Verwaltung augenscheinlich nicht mit allgemeinen Verfahrensweisen zum Abgleich der angemeldeten Steuerpflichtigen mit potentiellen Steuerzahlem vertraut, und auch die Form der Erfassung von Steuerpflichtigen (durch Steuernummer und Klassifikation) erscheint ungesichert. Aufgrund dieser Beobachtungen drängt sich der Schluß auf, daß die bisher zentralisierte Aktenführung reformbedürftig ist und das Institut der Kontrollmitteilungen verankert werden müßte.

In der Steuererhebung wird das Problem der Außenstände von der tansanischen Steuerverwaltung offenbar relativ gut beherrscht. In der Regel übersteigen die Zahlungseingänge jeweils die für das Jahr vorberechneten Soll-Beträge. Dennoch sind die Außenstände lt. Auskunft des IDT ungewöhnlich hoch, was angesichts der schleichenden Inflation einem stillschweigenden Verzicht des Staates auf Einnahmen gleichkommt. Maßnahmen zur Entwicklung und Verbesserung von Verfahren, die die Erhebung und ggf. Vollstreckung beschleunigen würden - einschl. der Etablierung und Durchsetzung von damit verbundenen Institutionen des materiellen Rechts wie Verzugszinsen und Säumniszuschlägen - wären daher angebracht, werden bisher jedoch nicht angewandt.

Unklar bleibt die Abgrenzung zwischen Amtsveranlagung und Betriebs- bzw. Außenprüfung, obgleich zwischen beiden eine grundsätzliche, wenn auch diffuse Unterscheidung zu bestehen scheint. Vor allem im Hinblick auf die Kapitalgesellschaften kommt es gelegentlich auch zu Außenprüfungen. Zwölf Beamte für Außenprüfungen waren im Einsatz; die 
Ergebnisse sind aber offensichtlich bisland unbefriedigend. ${ }^{25}$ Abweichend von der ImportUmsatzsteuer, die dem Zollverfahren unterliegt, wird die Inlands-Umsatzsteuer (sales tax) von einer Unterabteilung des ITD erfaßt. Hier gilt, daß jeder Steuerpflichtige monatliche Erklärungen abzugeben hat, die zugleich Angaben über die steuerbaren Wirtschaftsgüter einschl. ihrer Preise enthalten müssen. In der Praxis heißt dies, daß durchnumerierte Rechnungen in Kopie beigeheftet werden. Kontrollen finden in den steuerpflichtigen Betrieben und im Prinzip auch durch Straßenkontrollen der Marktstände und ähnlicher Kleinunternehmer in Randbereichen des informellen Sektors statt.

Will jemand als Steuerpflichtiger am sog. Ringsystem teilnehmen, benötigt er ein "registered dealer certificate". Das dabei zu durchlaufende Verwaltungsverfahren ist mit umfangreichen Aufzeichnungen über die Natur der Geschäftstätigkeit verbunden, die vermutlich viele Steuerpflichtige dazu verleitet, durch Falscherklärungen u.ä. Ziel und Zweck dieses Verfahrens möglichst zu unterlaufen. Dabei sind - wie bei der Verbrauchsbesteuerung auch sonst üblich - hohe Steuersätze und Beträge von der Steuerumgehung betroffen.

\section{Die Zollverwaltung (Customs and Sales Tax Department, CST)}

Die Hauptabteilung Zoll und Verkaufssteuer besteht in ihrer gegenwärtigen Form seit Juli 1982. Ihre organisatorische Neuordnung und räumliche Verbindung mit der Steuerverwaltung war das Ergebnis der Auflösung des alten East African Customs and Excise Department (Sitz: Nairobi) und geschah durch Vereinigung des alten Department of Customs mit dem Revenue Department. Trotz dieser formalen Integration bestanden die beiden Hauptabteilungen bis 1984 praktisch abgeschottet nebeneinander, jedoch unter Leitung eines für beide zuständigen Hauptabteilungsleiters.

Die in anderem Zusammenhang bereits erwähnten Reformmaßnahmen26 hatten ab 1981 als wesentliches Ergebnis die Schaffung einer Unterabteilung für Planung der Zollpolitik (Policy, Planning and Research Division), die allerdings ursprünglich in der Form einer übergreifenden Kommission verwirklicht werden sollte. Praktisch arbeitet diese Unterabteilung als besonderes Sekretariat des Hauptabteilungsleiters.

25 Interviewaussagen, UNDP-Projektmitarbeiter, Finanzministerium.

26 Die UNDP-Vorschläge haben ihren Niederschlag gefunden in einem vertraulichen Bericht des Finanzministeriums: Ministry of Finance, Economic Affairs and Planning, Report on Reorganisation of Department of Customs and Sales Tax, Daressalam, Juli 1986. Die früheren Vorschläge des IMF sahen vor allem eine Zusammenlegung des Revenue Department mit dem Income Tax Department vor. Da dies die verwaltungsmäßige Verbindung disparater Steuerarten bedeutet hätte, wurden die Vorschläge nach Kabinettsberatung zu den Akten gelegt. 
Insgesamt ist das CST innerhalb seines Aufgabenbereichs verantwortlich für die Anwendung sowohl der Steuer- und Abgabengesetze wie der Kontrollverordnungen. Neben Customs Code and Tariff sowie Sales Tax Act fallen die im Zusammenhang mit der Verkaufssteuer angeführten Nebensteuem in seine Zuständigkeit sowie auch die folgenden, z.Z. wenig ergiebigen Steuermaterien: Hotel Levy Act, Entertainment Tax Act, Land Ordinance and Road Toll Ordinance. Sowohl im Verwaltungsaufwand wie im Ertrag liegt der Schwerpunkt bei der steuerlichen Erfassung der einheimischen Manufaktur und bei den Import-/Exportabgaben (zusammen rd. $85 \%$ des Aufkommens). Das CST unterhält zu diesem Zweck Zollämter in den 21 Regionen, 32 Außenstellen sowie Hafenämter in drei See- und zwei Flughäfen.

Nach Maßgaben des UNDP-Beratungsprojekts ist mittlerweile eine interne Behördenstruktur etabliert worden, die sich unterhalb der Leitungsebene der Hauptabteilung (commissioner) in sieben Unterabteilungen (divisions) gliedert, von denen jede einem assistant commissioner untersteht. Davon sind fünf Unterabteilungen als sog. operational divisions unmittelbar mit Steuer- bzw. Zollaktivitäten betraut:

- Unterabteilung Zoll (Customs Division, CD)

- Unterabteilung Umsatz- und andere Steuern (Sales and other Taxes Division, STD)

- Unterabteilung Zollfahndung (Investigation Division)

- Unterabteilung Zoll- und Steuerprüungen (Audit and Inspection Division)

- Unterabteilung für Personalverwaltung und Rechnungsführung (Administration and Accounts Division)

Die beiden ersten Divisions teilen sich die eigentlichen Funktionen der Abgabenerhebung, und es ist eine Besonderheit der tansanischen Situation, daß die STD im Aufkommen im Vergleich zur Customs Division ein völlig überproportionales Gewicht erlangt hat und bislang beibehält. Der Grund dafür liegt in der jahrzehntelang untergeordneten Bedeutung des zollpflichtigen Außenhandels, der durch staatlichen Lizensierungszwang, Devisenzwangsbewirtschaftung und andererseits weitreichende Zollfreistellung der staatlichen Importe zurückgedrängt wurde.

Bis 1986 zeigte das Zollgebührenaufkommen keine der Wirtschaftsentwicklung angepaßte Elastizität und stagnierte um $7 \%$ der gesamten Steuereinnahmen (= rd. 1,5\% des BIP). Erst seit der auch außenwirtschaftlich relevanten Kursänderung ab 1987 im Zusammenhang mit dem Economic Recovery Programme gewinnt der Zoll als Komponente der fiskalischen Abgaben wieder an Bedeutung (ca. $14 \%$ des Steueraufkommens 1989/90), liegt aber noch immer weit zurück gegenüber den inländischen Verbrauchssteuern (56\%) und den Lohn- und Einkommensteuem (25\%).

Aus dem gleichen Zusammenhang einer politisch bedingten Minderbewertung der Zollabgaben im Rahmen des tansanischen Importsubstitutionsmodells erklärt sich, daß der Zoll- 
verwaltung und nicht dem ITD im Verlauf der Reorganisation die Verwaltung der Verkaufssteuer überantwortet wurde. Auch in Afrika benutzen nur wenige Länder ihre Zollverwaltungen zur Erhebung der allgemeinen Verkaufssteuer. Ein zusätzlicher Grund ist darin zu suchen, daß zuvor die frühere excise tax mit der Verkaufssteuer zusammengelegt worden war und die letztere in ihrer sehr differenzierten Gestaltung der Warenklassifikation den typischen Zoll- und Akzise-Aufgaben ähnelt.

Schwächen des Zollverwaltungsverfahrens liegen in erster Linie bei der Wareninspektion, der Zollbewertung der Waren und der Sicherung der Lagerkapazitäten. Die Inspektion am Objekt wird größtenteils jüngeren, unerfahrenen Nachwuchskräften überantwortet, die "kräftig anpacken" können, aber noch wenig Erfahrung darin haben, wonach eigentlich zu suchen ist. Die Container-Inspektion (auf freien Lagerhöfen) ist besonders defizient und zieht hohe Schwundraten durch Diebstahl nach sich.

Als Folgeerscheinung unprofessioneller Zollinspektion sind zwei Probeme für die tansanische Fiskalverwaltung gegenwärtig kennzeichnend: einerseits weitverbreitete Unterbewertung von Importwaren, die dann auf dem Parallelmarkt vom Importeur mit unbesteuertem Gewinn verkauft werden; andererseits aber auch unkontrollierte Höherbewertungen der Importwaren zurn Zweck der Kapitalverschiebung ins Ausland.

\section{Schlußfolgerungen}

Wie der Uberblick über die tansanische Finanzpolitik zeigte, hat sich die Lage der Haushaltsfinanzierung über die Jahre kontinuierlich verschlechtert. Die Haushaltssteuerung dieses auf self-reliance und Handlungsautonomie eingeschworenen Landes ist bis in die Gegenwart in ungewöhnlich hohem Umfang von ausländischen Hilfszahlungen abhängig geblieben, die als Haushaltssubventionen wirken, regelmäßig den gesamten Investitionshaushalt abdecken und ihn in einzelnen Jahren (so 1990) sogar deutlich überschritten. Abgesehen von einer Reihe von Außeneinflüssen - wie die Entwicklung der Terms of Trade und der Energiepreis-Krise von 1978 - waren hierfür maßgeblich die Verlustsituation im staatsnahen Wirtschaftssektor und die durch das gewählte Steuersystem verursachte Inelastizität der Besteuerungsbasis.

In dem von abnehmender Effizienz gekennzeichneten Sektor der parastatals waren es vor allem die Vermarktungskörperschaften des Staatsmonopols (crop authorities), die durch erratische Verluste die staatliche Defizitfinanzierung ausgelöst und Tansanias makroökonomische Probleme mitverursacht haben. 27 Eigene Maßnahmen des Landes im Bereich der Ausgaben- und Fiskalpolitik waren demgegenüber bisher nur von geringem Einfluß im

27 So auch C. G. Kahama u.a., a.a.O., S. $152 \mathrm{f}$. 
Sinne eines Ausgleichs der Ausgaben-/Einnahmenstruktur und Budget-Sanierung. Bis in die späten 80er Jahre ließ die Steuerpolitik eine Vernachlässigung entwicklungsökonomischer Parameter erkennen, die eine Aktivierung der einheimischen Wirtschaftsaktivitäten über die Wiederanknüpfung des Handelsaustausches mit dem Ausland erlaubt hätten. Statt dessen wurde die Importsubstitutionspolitik bis über ihre inhärenten Grenzen hinaus fortgeführt, mit entsprechend rezessiven Folgeerscheinungen und Produktivitätseinbußen. Angesichts der so entstandenen Wirtschaftsstruktur stützt sich die Fiskalpolitik überwiegend auf die indirekte Besteuerung von Waren und Dienstleistungen (Verbrauchssteuern, insbesondere Umsatzsteuer in der Form der tansanischen Sales Tax von 1969). Diese bleibt mit rd. $60 \%$ am gesamten Einnahmevolumen die wichtigste Einnahmequelle des Staates, gefolgt von Einkommen-/Körperschaftsteuem und Kapitalertragssteuem, die zusammen nur etwas über $25 \%$ des Steueraufkommens erbringen (vgl. Tab. 2). Als Einkommensteuer-Zahler leisten rd. 70.000 natürliche Personen nur knapp $10 \%$ des gesamten Aufkommens.

Eine strukturelle Fehlkonzeption der einkommensteuerlichen Behandlung von Privatpersonen liegt in der durch die Reform von 1974 verschärften Progression. Durch sie war auch die Zusammenveranlagung von Ehepaaren abgeschafft worden, mit der Folge entsprechend erhöhter Steuerbelastung. Bis 1989 war hier infolge der Inflation eine geradezu groteske Verzerrung eingetreten: Die Freigrenze, die sich am staatlich fixierten Mindestlohn orientiert, lag bei monatlich 800 TSh und war damit auf einen Gegenwert von DM 11 gesunken, und bereits bei einem Monatseinkommen von 4.000 TSh (=ca. DM 59) war der marginale Steuersatz von $50 \%$ erreicht. Personalaufstockungen in der Finanzverwaltung hatten zu einer verbesserten Erfassung der Steuerpflichtigen geführt, die aber ganz überwiegend auf die leicht erschließbare Gruppe der Industriearbeiterschaft und Angestellten im Hauptstadtbereich begrenzt blieb. Die gesamte Einkommen-Besteuerung hat dadurch nunmehr eine ungleiche Belastung des städtischen Sektors gegenüber dem agrarischen Hinterland nach sich gezogen, und hier ist die Besteuerungsgrenze seit Mitte der 80er Jahre eindeutig erreicht - eine disfunktionale Entwicklung, die ganz wesentlich zum Ausweichen der Steuerbürger in (steuerlich bisher nicht erfaßte) informelle Wirtschaftsaktivitäten beiträgt.

Während der Komplex der Einkommensteuern somit im Verlauf der Zeit einen abnehmenden Anteil am Brutto-Inlandsprodukt 28 und einen relativ absinkenden Beitrag zum Staatshaushalt geleistet hat, hat die Abhängigkeit des tansanischen Fiskus von der indirekten Besteuerung immer mehr zugenommen. Die damit im gegenwärtigen System angelegte Tendenz zur Regressivität, d.h. einer überproportionalen Belastung unterer Einkommensgruppen, wird nur ausgeglichen durch die bisher aus politischen und administrativen Grün-

28 Der Anteil des Aufkommens der individuellen Einkommensteuer am BIP ging von 2,4 \% (1979) auf $1,9 \%$ (1987) zurück, und der der Körperschaftsteuer von $4 \%$ auf 2,9\%, während der Anteil der Umsatzsteuer sich von 8,3\% auf $11 \%$ des BIP erhöhte (Quelle: Berechnung aufgrund: Tanzania: Statistical Survey, Daressalam, Govemment Printer 1989). 
den in Kauf genommene Vernachlässigung eines wesentlichen Sektors des BIP - nämlich der Subsistenz- und Schattenwirtschaft - und durch die Luxus-Importbesteuerung in extremer Höhe.

\begin{abstract}
Abgesehen von den nominal, nicht inflationsbereinigt erzielten Zuwachsraten der Kassenzuflüsse ist die Besteuerungsbasis in den vergangenen Jahren real zurückgegangen. Im gleichen Maße sanken Verkaufsvorgänge, Importe und Gewinne während der Handelsabschottung und im Verlauf der Wirtschaftskrise ab. Dies hat die Regierung 1984 zur Einführung einer "development levy" veranlaßt, einer Art Kopfsteuer oder poll tax, die unterschiedslos von allen Erwachsenen eingezogen wird,29 sich aber ihrerseits dann fiskalisch als Schlag ins Wasser erwiesen hat.
\end{abstract}

Erst seit der wirtschaftlichen Offnung und dem zaghaften Anstieg des Wirtschaftswachstums sind wieder Anzeichen für eine Erweiterung der Besteuerungsbasis erkennbar, was sich zunächst bei den Einfuhrzöllen und im erhöhten Umsatzsteueraufkommen niederschlägt.

Auch weiterhin aber, und zumindest auf mittlere Sicht weiterwirkend, orientieren sich die steuerpolitischen Zielvorstellungen der tansanischen Regierung an einer grundlegenden Beibehaltung der etablierten Steuerstrukturen, wenn auch unter Anpassung der bisher starren Steuersätze, unter Einsatz effizienterer Erfassungsverfahren und bei erhöhter administrativer Leistungsfähigkeit. Dies wird bedeuten, daß das Schwergewicht auf Verbrauchssteuem grundsätzlich beibehalten wird. Angesichts der bestehenden Vielfalt von knapp 30 Umsatzsteuersätzen wurde seit 1989 über eine schrittweise Vereinfachung und Herabsetzung der Spitzensätze und über die Schaffung von Umsatzsteuer-Kreditkonten beraten. Von diesen Maßnahmen erwartet man sich eine Erweiterung der Besteuerungsgrundlagen und die Möglichkeit einer gerechteren Anwendung der Verbrauchssteuem. Ein Politikum ist die damit implizit angestrebte Einbeziehung der Schattenwirtschaft, d.h. insbesondere des informellen Straßen- und Markthandels, und dies läßt die Reformentschlossenheit immer wieder erlahmen.

29 Diese Steuer wird auf der lokalen Ebene von den zuständigen Büros der Staatspartei eingezogen; $17 \%$ des Aufkommens sollen den Kommunalhaushalten zugeführt werden. Steuerpolitisch und fiskalisch ist diese Maßnahme als charakteristischer Fehlschlag zu bewerten. Zum einen ist der Kopfsteuerbetrag mit TSh 250 so gering bemessen (und nicht ohne politische Erschütterungen an die Inflationsentwicklung anzupassen), daß sein Ertrag von ursprünglich etwa DM 3,70 bis 1990 auf DM 1,27 pro Kopf zusammengeschmolzen war, was kaum die Verwaltungskosten decken kann. Zum anderen sind die Parteizellen in erster Linie an der Festigung der Parteibasis interessiert und verhalten sich entsprechend "großzügig" bei der Steuererhebung. Die tatsächliche Erfassungsquote wird auf unter $50 \%$ geschätzt. 
Im Bereich der Einkommensteuer und speziell der Körperschaftsteuer wird erwartet, daß die bereits vollzogene Absenkung der Spitzensteuerbelastung, gegebenenfalls auch die Lockerung der Freigrenzen und die Anpassung der Kapitalertragsteuer an die Inflationsgegebenheiten gesamtwirtschaftlich wirkende Leistungsimpulse freisetzen, die - zusammen mit administrativen und organisatorischen Verbesserungen der Steuererfassung - das Aufkommen überproportional erhöhen sollten.

Bei diesem Rückblick auf die Fiskalpolitik Tansanias und ihre administrative Bewältigung während der vergangenen Jahrzehnte - und speziell in den 80er Jahren - wird deutlich, daß die politische Führung des Landes bewußt weitgehend auf inländische Kapitalschaffung, industrielle Modemisierungsinvestitionen und außenwirtschaftliche Dynamik mit Einbindung in den Weltmarkt verzichtet hat. Die zunehmende Abhängigkeit von internationaler Entwicklungshilfe hat zwar lange Zeit das Investitionsbudget über Wasser gehalten, aber ebenfalls keine tragfähige moderne Wirtschaftsstruktur hervorbringen können.

Wenn seit Ende der 80er Jahre eine Umkehr aus der Sackgasse gesucht wird, so fällt die neue Bereitschaft zur Modemisierung durch Kooperation mit ausländischen Investoren nun in eine Zeit weltweiten Investitionshungers und der Ressourcenverknappung. Wie sich jetzt zeigt, hat Tansania entscheidende Jahrzehnte verpaßt, in denen internationales Investitionskapital verfügbar und bereit gewesen wäre, die relativen Standortvorteile des Landes innerhalb Afrikas zu nutzen. Maßnahmen, wie sie inzwischen im Zoll- und Steuerrecht und in der Finanzverwaltung eingeleitet worden sind, lassen den Willen zu pragmatischer Problemorientierung erkennen. Angesichts der weltpolitischen Entwicklung und der dadurch verstärkten Marginalisierung Afrikas werden sie jedoch vermutlich für einige Zeit Teil einer neuen Form wirtschaftlicher Self-reliance bleiben müssen. 


\section{ABSTRACTS}

\section{International Trade Rules and Environmental Protection}

\section{By Rolf Knieper and Gerd Winter}

Protectionism as well as liberalism in international trade have in the past been debated as different ways of how to create wealth and, by implication, how to exploit nature most effectively. As to exploitation of nature they have indeed been effective, the first due to its tragic sliding into technological backwardness, the second due to its power of fostering growth. Meanwhile, nature has been realized to be a finite resource. Environmental protection is being recognized as a new goal against which protectionism and liberalism are to be measured. The authors develop a conceptual frame-work for such analysis and apply it to the major conventions on international trade. They distinguish between conventions which primarily foster growth by either providing a general legal infrastructure (like e.g. contract law and patent law) or by removing specific trade restrictions, on the one side, and conventions which primarily strive for environmental protection by directly restricting trade. Trade related conventions basically regard environmental protection as an obstacle, environment related conventions see trade as a nuisance. Both types have started with a concept of internationally supervised national (environmental) protectionism but forcefully evolve towards supranational structures.

Fiscal Policy and Tax Administration in Tanzania during the "Lost Decade" of Economic Development

\section{By Ulrich Fanger}

In the course of several decades of self-imposed economic Self-Reliance, Tanzania permitted her fiscal and budgetary policies to slip into increasing dependency on foreign financial contributions in order to cover the widening budgetary deficits. While obviously due to constant govemmental over-spending on subsidies, this development had at its source the diminishing fiscal revenue of the country and its shrinking tax base.

The article is based on the results of a study of the Tanzanian tax system and the public administration involved in taxation and customs operations. They show that taxation policy developed a strong bias in favour of indirect taxes (purchase and sales taxes, customs 
duties) as against direct taxation, thereby giving the entire tax system a definitely regressive slant. Income taxation being based on prohibitively high progressive rates, its administrative application became uneven and inefficient. At the same time, the country's commercial isolation virtually wiped out customs duties as the major source of revenue from indirect levies. The study emphasizes the effects which the gradual systemic distortion had upon the administrative side. Assessment and tax collection deteriorated due to staffing problems caused by below-subsistence level emoluments. The effects are still visible in the age gap and loss of experienced personnel that are a consequence of high fluctuation rates out of the public service.

The paper finally examines the possibilities and prospective consequences of the step-bystep fiscal policy revision that has been implemented since 1987, as a collateral to the two successive Economic Recovery Programmes agreed upon with the World Bank and IMF.

\section{Multi-Partyism versus Democracy in Southern Africa: "Whither Lesotho"}

\section{By Khabele Matlosa}

In many African countries, the debate on multi-partyism has become one of the most important and most ardently disputed issues in constitutional law and on the political scene. Whereas Western countries favor the concept of multy-partyism without exception, the African debate is characterized by a more diversified and a more critical view. The African dispute is taking into consideration the situation of the political systems involved and the legal structure provided within the specific countries concerned. And it does not separate politics and constitutional law artificially.

The case study on multi-partyism in Lesotho may be exemplary in this sense. It is, at the same time, uncomparable and unique, as Lesotho economic, social and political background does not compare to many other African countries.

The article begins with contrasting definitions of the concepts of multi-partyism and democracy in a Western and in an African setting. It then continues with a synopsis of the African democracy debate past and present. Finally, the author provides a detailed analysis of democracy in Lesotho itself. He concludes that the "military democracy" Lesotho is facing may not lead to true multi-partyism in the near future. Nevertheless, the options and possibilities that do exist for Lesotho are reviewed and critically discussed. 